\title{
Lineability within Peano Curves, Martingales, and Integral Theory
}

\author{
Artur Bartoszewicz $\left(\mathbb{D}\right.$, Marek Bienias $\mathbb{D}^{D}$, and Szymon Gląb \\ Institute of Mathematics, Lodz University of Technology, Wólczańska 215, 90-924 Łódź, Poland \\ Correspondence should be addressed to Marek Bienias; marek.bienias@p.lodz.pl
}

Received 16 October 2018; Accepted 12 November 2018; Published 4 December 2018

Academic Editor: Eva A. Gallardo Gutiérrez

Copyright (C) 2018 Artur Bartoszewicz et al. This is an open access article distributed under the Creative Commons Attribution License, which permits unrestricted use, distribution, and reproduction in any medium, provided the original work is properly cited.

\begin{abstract}
This paper is devoted to give several improvements of some known facts in lineability approach. In particular, we prove that (i) the set of continuous mappings from the unit interval onto the unit square contains a closed, $\mathfrak{c}$-semigroupable convex subset, (ii) the set of pointwise convergent martingales $\left(X_{n}\right)_{n \in \mathbb{N}}$ with $E\left|X_{n}\right| \longrightarrow \infty$ is $\mathfrak{c}$-lineable, (iii) the set of martingales converging in measure but not almost surely is $\mathfrak{c}$-lineable, (iv) the set of sequences $\left(X_{n}\right)_{n \in \mathbb{N}}$ of independent random variables, with $E X_{n}=0$, $\sum_{n=1}^{\infty} \operatorname{var} X_{n}=\infty$, and the property that $\left(X_{1}+\cdots+X_{n}\right)_{n \in \mathbb{N}}$ is almost surely convergent, is $\mathfrak{c}$-lineable, (v) the set of bounded functions $f:[0,1] \times[0,1] \longrightarrow \mathbb{R}$ for which the assertion of Fubini's Theorem does not hold is consistent with ZFC 1-lineable (it is not 2-lineable), (vi) the set of unbounded functions $f:[0,1] \times[0,1] \longrightarrow \mathbb{R}$ for which the assertion of Fubini's Theorem does not hold (with infinite integral allowed) is $\boldsymbol{c}$-lineable but not $\boldsymbol{c}^{+}$-lineable.
\end{abstract}

\section{Introduction}

We start with a standard introduction to the idea of lineability (similar openings can be found, for example, in our paper with T. Natkaniec (see [1]) or in the papers of other authors mentioned below). For more than a decade now, many mathematicians have been looking at the largeness of some sets by constructing algebraic structures inside them. This approach is called lineability. A comprehensive description of this concept as well as numerous examples and some general techniques can be found in the book [2] and references therein. Following R. Aron, A. Bartoszewicz, S. Głąb, V. Gurariy, D. Pérez-García, and J.B. Seoane-Sepúlveda, let us recall the following notion.

Definition 1. Let $\kappa$ be a cardinal number.

(1) Let $\mathscr{L}$ be a vector space and $A \subseteq \mathscr{L}$. We say that $A$ is $\kappa$-lineable if $A \cup\{0\}$ contains a $\kappa$-dimensional subspace of $\mathscr{L}$.

(2) Let $\mathscr{L}$ be a semigroup and $A \subseteq \mathscr{L}$. We say that $A$ is $\kappa$-semigroupable if $A$ contains a $\kappa$-generated subsemigroup of $\mathscr{L}$.
This paper is devoted to the improvements of some known facts: in semigroupability (Section 2) and lineability in probability theory (Section 3). In Sections 4 and 5 we present some new results in probability and integral theory. Along this paper we shall use standard set theoretical notation and notions. In particular, we identify an ordinal number $\alpha$ with the set of all ordinals $\beta<\alpha$. Cardinal numbers are those ordinals $\alpha$ which are not equipotent with any $\beta<\alpha$ and by $\alpha^{+}$we denote the cardinal successor of a cardinal number $\alpha$. We use standard notation, i.e., $\mathbb{N}=\{1,2,3, \ldots\}$.

\section{Peano Curves and Semigroupability}

In the $19^{\text {th }}$ century, inspired by the result that the unit interval $[0,1]$ is of the same cardinality as the unit square $[0,1]^{2}, \mathrm{G}$. Peano constructed (see [3]) a continuous onto mapping $F$ : $[0,1] \longrightarrow[0,1]^{2}$. For topological spaces $X, Y$ let us denote the set of all continuous surjections from $X$ to $Y$ by $\mathscr{C} \mathcal{S}(X, Y)$. In [4], N. Albuquerque proved that for every pair $n, m \in \mathbb{N}$ the set $\mathscr{C} \mathcal{S}\left(\mathbb{R}^{m}, \mathbb{R}^{n}\right)$ is c-lineable.

Let $\mathbb{q}=[0,1]$. In this section we are interested in the set

$$
\mathscr{C} \mathcal{S}\left(\square, \square^{2}\right)=\left\{f: \llbracket \longrightarrow \mathbb{\square}^{2}: f \text { is cont. and } f[\square]=\rrbracket^{2}\right\} \text {. }
$$


L. Bernal-González, M.C. Calderón-Moreno, and J. PradoBassas proved in [5] that the set $\mathscr{C} \mathcal{S}\left(\square, \rrbracket^{2}\right)$ is semigroupable; i.e., it contains $\omega$-generated semigroup (with respect to coordinate-wise multiplication). Our goal is to prove the following.

Theorem 2. There exists a closed convex set $\mathscr{X} \subseteq \mathscr{C} \mathcal{S}\left(\mathbb{\square}, \mathbb{q}^{2}\right)$ such that it is $\mathbf{c}$-semigroupable; i.e., $\mathscr{X}$ contains a $\mathfrak{c}$-generated semigroup.

Proof. Let us fix a function $F \in \mathscr{C} \mathcal{S}\left([0,1 / 2], \square^{2}\right)$ (its construction is a trivial modification of Peano's example) and let $\mathscr{A} \subseteq[0,1]^{\mathbb{N}}$ be a set of cardinality $c$ of sequences that converge to 0 and are free generators (see [6]). Let $\mathscr{F}=\left\{F_{x}: x \in \mathscr{A}\right\}$,

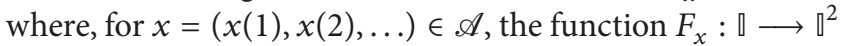
is defined by the formula

$$
F_{x}(t)= \begin{cases}F(t), & \text { when } t \in\left[0, \frac{1}{2}\right] \\ (t, x(n)), & \text { when } t=1-\frac{1}{n+2} \text { for } n \in \mathbb{N} \\ (1,0), & \text { when } t=1 \\ \text { affine, } & \text { elsewhere, }\end{cases}
$$

(by affine we mean line segments in the square $\mathbb{q}^{2}$ that continuously connect points $F(1 / 2)$ with $(2 / 3, x(1))$ and $(1-$ $1 /(n+2), x(n))$ with $(1-1 /(n+3), x(n+1))$ for $n=1,2, \ldots)$. Clearly $F_{x}$ is continuous for every $x \in \mathscr{A}$. Let $\mathcal{y}$ be a semigroup generated by $\mathscr{F}$; i.e., $\mathscr{Y}=\left\{F_{x_{1}}^{k_{1}} \cdot \ldots \cdot F_{x_{n}}^{k_{n}}\right.$ : $\left.x_{1}, \ldots, x_{n} \in \mathscr{A}, k_{1}, \ldots, k_{n} \in \mathbb{N}, n \in \mathbb{N}\right\}$. We will show that $\mathscr{X}=\overline{\operatorname{conv}(\mathscr{Y})}$ (the closure of the convex hull of $\mathscr{Y}$ ) has the desired properties. It is easy to see that since $\mathscr{A}$ is a set of free generators, the set $\mathscr{F}$ is algebraically independent. Hence, $\mathscr{Y}$ is a $\mathbf{c}$-generated semigroup.

Moreover, $\operatorname{conv}(\mathscr{Y}) \subseteq \mathscr{C} \mathcal{S}\left(\square, \rrbracket^{2}\right)$. Indeed, let $f \in \operatorname{conv}(\mathcal{Y})$. Then there are $n \in \mathbb{N}, f_{1}, \ldots, f_{n} \in \mathcal{Y}$ and $\alpha_{1}, \ldots, \alpha_{n} \in(0,1]$ such that $\sum_{i=1}^{n} \alpha_{i}=1$, with $f(t)=\sum_{i=1}^{n} \alpha_{i} f_{i}(t)$ for $t \in \mathbb{\square}$. Clearly $f$ is continuous. It is easy to see that, by the definition of the functions from $\mathscr{F}$, there exist $k_{1}, \ldots, k_{n} \in \mathbb{N}$ such that $f \mid[0,1 / 2]=\sum_{i=1}^{n} \alpha_{i} F^{k_{i}}$. To show that $f \in \mathscr{C} \mathcal{S}\left(\square, \mathbb{Q}^{2}\right)$, it is enough to prove that $f[[0,1 / 2]]=\mathbb{\square}^{2}$. Let $(a, b) \in \mathbb{\square}^{2}$ and let $P: \square \longrightarrow$ \ be defined by $P(t)=\sum_{i=1}^{n} \alpha_{i} x^{k_{i}}$. Since $P(0)=0$ and $P(1)=1$, the function $P$ is onto and there exist $x, y \in \llbracket$ such that $P(x)=a$ and $P(y)=b$. Moreover, as $F \in \mathscr{C} \mathcal{S}\left([0,1 / 2], \mathbb{1}^{2}\right)$, there is $t \in[0,1 / 2]$ with $F(t)=(x, y)$ and then $f(t)=P(F(t))=(a, b)$ (for a function $F=\left(F_{1}, F_{2}\right)$ :

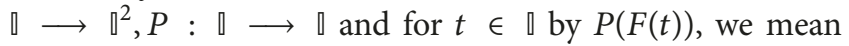
$\left.\left(P\left(F_{1}(t)\right), P\left(F_{2}(t)\right)\right) \in \mathbb{\natural}^{2}\right)$. Hence, $f \in \mathscr{C} \mathcal{S}\left(\mathbb{\square}, \mathbb{q}^{2}\right)$.

Finally, let us observe that the limit of any convergent sequence from $\mathscr{C} \mathcal{S}\left(\square, \mathbb{Q}^{2}\right)$ is still in $\mathscr{C} \mathcal{S}\left(\square, \mathbb{Q}^{2}\right)$. Indeed, let $\left(g_{n}\right)_{n \in \mathbb{N}}$ be a sequence of elements of $\mathscr{C} \mathcal{S}\left(\mathbb{\square}, \mathbb{\square}^{2}\right)$ that converges

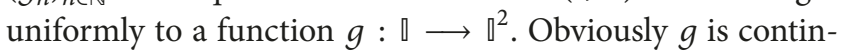
uous. Moreover, for any point $w \in \mathbb{q}^{2}$ and any $n \in \mathbb{N}$ there is $x_{n} \in \mathbb{\text { with }} g_{n}\left(x_{n}\right)=w$. We may assume (by compactness of 『) that $\left(x_{n}\right)_{n \in \mathbb{N}}$ is convergent to some point $x_{0} \in \mathbb{\square}$. It is easy to see that $\lim _{n \rightarrow \infty} g_{n}\left(x_{n}\right)=g\left(x_{0}\right)$ so $g\left(x_{0}\right)=w$. Hence, $g$ is also surjective. Thanks to this observation, we have proved that $\mathscr{X}=\overline{\operatorname{conv}(\mathscr{Y})}$ is a closed, convex, and $\mathfrak{c}$-semigroupable subset of $\mathscr{C} \mathcal{S}\left(\square, \mathbb{\square}^{2}\right)$.

\section{Lineability within Martingales Theory}

This section is devoted to some lineability problems in the martingales setting. Let us start with a short introduction to the probability and martingales theory. Till the end of the section let $(\Omega, \mathcal{\delta}, P)$ be a probability space (i.e., $\Omega$ is a nonempty set, $\mathcal{\delta}$ is a $\sigma$-algebra of subsets of $\Omega$, and $P: \mathcal{S} \longrightarrow$ $[0,1]$ is a probability measure). A function $X: \Omega \longrightarrow \mathbb{R}$ is called a random variable, whenever it is $\mathcal{S}$-measurable (i.e., $X^{-1}[B] \in \mathcal{S}$ for every Borel $B \subseteq \mathbb{R}$ ); its expected value is computed as follows:

$$
E X=\int_{\Omega} X d P
$$

and its variance is as follows:

$$
\operatorname{var} X=E X^{2}-(E X)^{2} \text {. }
$$

There are different notions of convergence of a sequence of random variables. We recall those that will be needed later.

Definition 3. Let $X$ and $X_{n}(n \in \mathbb{N})$ be random variables. We say that the sequence $\left(X_{n}\right)_{n \in \mathbb{N}}$

(1) is pointwise convergent to $X$, whenever $X_{n}(t) \longrightarrow$ $X(t)$ for every $t \in \Omega$,

(2) is convergent almost everywhere to $X$, whenever $X_{n}(t) \longrightarrow X(t)$ almost surely, i.e.,

$$
P\left(\left\{t \in \Omega: X_{n}(t) \longrightarrow X(t)\right\}\right)=1,
$$

(3) is convergent in measure to $X$, whenever

$$
\forall_{\varepsilon>0} \quad P\left(\left\{t \in \Omega:\left|X_{n}(t)-X(t)\right| \geq \varepsilon\right\}\right) \longrightarrow 0 .
$$

It is clear that pointwise convergence implies convergence almost surely and it implies convergence in measure. Moreover, none of these implications can be reversed in general.

Definition 4. Let $X: \Omega \longrightarrow \mathbb{R}$ be a random variable with finite expected value and let $\mathscr{H} \subseteq \mathcal{S}$ be a $\sigma$-algebra. The conditional expectation of $X$ with respect to $\mathscr{H}$, denoted by $E(X \mid \mathscr{H})$, is any $\mathscr{H}$-measurable function $\Omega \longrightarrow \mathbb{R}$ that satisfies

$$
\int_{H} E(X \mid \mathscr{H}) d P=\int_{H} X d P
$$

for every $H \in \mathscr{H}$.

It is known that the conditional expectation of $X$ with respect to $\mathscr{H}$ is defined uniquely up to a set of measure zero. In the sequel the following properties of conditional expectation will be useful (see [7]).

\section{Remark 5.}

(1) Let $X, Y: \Omega \longrightarrow \mathbb{R}$ be random variables, let $\mathscr{G} \subseteq \mathcal{S}$ be a $\sigma$-algebra, and assume that $X$ is $\mathscr{G}$-measurable. If $X$ and $X Y$ have finite expected values, then

$$
E(X Y \mid \mathscr{G})=X E(Y \mid \mathscr{G}) \text {, almost surely. }
$$


(2) Let $X: \Omega \longrightarrow \mathbb{R}$ be a random variable and $\mathscr{G}_{1} \subseteq \mathscr{G}_{2} \subseteq$ $\mathcal{S}$ are $\sigma$-algebras. Then

$$
E\left(E\left(X \mid \mathscr{G}_{2}\right) \mid \mathscr{G}_{1}\right)=E\left(X \mid \mathscr{G}_{1}\right) .
$$

(3) Let $X: \Omega \longrightarrow \mathbb{R}$ be a random variable and let $\mathscr{G} \subseteq \mathcal{S}$ be a $\sigma$-algebra such that $\sigma(X)$ and $\mathscr{G}$ are independent (i.e., for all $A \in \sigma(X)$ and $B \in \mathscr{G}$ we have $P(A \cap B)=$ $P(A) \cdot P(B)$, where $\sigma(X)$ is the smallest $\sigma$-algebra with respect to which $X$ is measurable). Then

$$
E(X \mid \mathscr{G})=E X
$$

i.e., it is a constant random variable.

Definition 6. We say that a sequence $\left(\mathscr{F}_{n}\right)_{n \in \mathbb{N}}$ of $\sigma$-algebras forms a filtration whenever

$$
\mathscr{F}_{1} \subseteq \mathscr{F}_{2} \subseteq \cdots \subseteq \mathcal{S} .
$$

Let us state the definition of a martingale.

Definition 7. Let $\left(\mathscr{F}_{n}\right)_{n \in \mathbb{N}}$ be a filtration and $\left(X_{n}\right)_{n \in \mathbb{N}}$ be a sequence of random variables. We say that $\left(X_{n}\right)_{n \in \mathbb{N}}$ is a martingale adapted to the filtration $\left(\mathscr{F}_{n}\right)_{n \in \mathbb{N}}$ if for every $n \in \mathbb{N}$

(1) $X_{n}$ is $\mathscr{F}_{n}$-measurable,

(2) $E\left|X_{n}\right|<\infty$,

(3) $E\left(X_{n+1} \mid \mathscr{F}_{n}\right)=X_{n}$ almost everywhere.

Remark 8. The set of all martingales adapted to a fixed filtration forms a real vector space (with respect to natural addition and scalar multiplication).

In [8], the authors proved the following theorem:

Theorem 9 (Thm. 1, [8]). There is a probability space in which the set of pointwise convergent sequences of random variables $\left\{X_{n}: n \in \mathbb{N}\right\}$ with $E\left|X_{n}\right| \longrightarrow \infty$ is $\omega$-lineable.

Our goal is to state a more general result than the above in the setting of martingales.

Theorem 10. Let $(\Omega, \mathcal{S}, P)$ be a probability space such that there is infinitely many pairwise disjoint sets of positive measures. Then there exist a filtration $\left(\mathscr{F}_{n}\right)_{n \in \mathbb{N}}$ and $\mathfrak{c}$-dimensional vector space $\mathbb{V}$ of martingales adapted to the filtration $\left(\mathscr{F}_{n}\right)_{n \in \mathbb{N}}$ such that for every nonzero $X=\left(X_{n}\right)_{n \in \mathbb{N}} \in \mathbb{V}$ we have that $\left(X_{n}\right)_{n \in \mathbb{N}}$ is pointwise convergent but $E\left|X_{n}\right| \longrightarrow \infty$.

Proof. Let $A_{1}, A_{2}, \ldots, B_{1}, B_{2}, \ldots \in \mathcal{S}$ be a partition of $\Omega$ (i.e., $\Omega$ is a disjoint union of all $A_{i}$ 's with all $B_{i}$ 's) such that $P\left(A_{i}\right), P\left(B_{i}\right)>0$ for all $i \in \mathbb{N}$. Let $\mathscr{F}_{n}=\sigma\left(A_{1}, \ldots, A_{n}\right.$, $\left.B_{1}, \ldots, B_{n}\right)$, for $n \in \mathbb{N}$. Clearly $\left(\mathscr{F}_{n}\right)_{n \in \mathbb{N}}$ forms a filtration. For $\alpha \in(0,1)$ let us define $X^{(\alpha)}=\left(X_{n}^{(\alpha)}\right)_{n \in \mathbb{N}}$ in the following way: for $t \in \Omega$

$$
X_{n}^{(\alpha)}(t)= \begin{cases}\frac{1}{k^{\alpha} P\left(A_{k}\right)}, & \text { when } t \in A_{k}, \text { for } k \leq n \\ \frac{-1}{k^{\alpha} P\left(B_{k}\right)}, & \text { when } t \in B_{k}, \text { for } k \leq n \\ 0 & \text { elsewhere. }\end{cases}
$$

Observe that $X^{(\alpha)}$ is a martingale adapted to the filtration $\left(\mathscr{F}_{n}\right)_{n \in \mathbb{N}}$. Indeed, for any $n \in \mathbb{N}$,

(1) $X_{n}^{(\alpha)}$ is an $\mathscr{F}_{n}$-measurable;

(2) $E\left|X_{n}^{(\alpha)}\right|=2 \sum_{k=1}^{n}\left(1 / k^{\alpha}\right)<\infty$;

(3) as for $M=\bigcup_{k=1}^{n}\left(A_{k} \cup B_{k}\right)$ we have that $\int_{\Omega \backslash M} X_{n+1}^{(\alpha)}=0$ and $\left.X_{n+1}^{(\alpha)}\right|_{M}=\left.X_{n}^{(\alpha)}\right|_{M}$, so for every $C \in \mathscr{F}_{n}$ we have that $\int_{C} X_{n+1}^{(\alpha)}=\int_{C} X_{n}^{(\alpha)}$. Hence, $E\left(X_{n+1}^{(\alpha)} \mid \mathscr{F}_{n}\right)=X_{n}^{(\alpha)}$.

Let $\mathbb{V}=\operatorname{span}\left\{X^{(\alpha)}: \alpha \in(0,1)\right\}$. Then $\mathbb{V}$ is a vector space of martingales adapted to the filtration $\left(\mathscr{F}_{n}\right)_{n \in \mathbb{N}}$. Clearly its dimension equals $\mathfrak{c}$ as $\left\{X^{(\alpha)}: \alpha \in(0,1)\right\}$ is linearly independent. We will show that $\mathbb{V}$ has the desired properties. Let $X=$ $\left(X_{n}\right)_{n \in \mathbb{N}} \in \mathbb{V}$ be a nonzero martingale of the form $\left(X_{n}\right)_{n \in \mathbb{N}}=$ $\left(\sum_{i=1}^{m} c_{i} X_{n}^{\left(\alpha_{i}\right)}\right)_{n \in \mathbb{N}}$ for some $m \in \mathbb{N}, c_{1}, \ldots, c_{m} \in \mathbb{R} \backslash\{0\}$ and $0<\alpha_{1}<\alpha_{2}<\cdots<\alpha_{m}<1$. It is easy to see that $\left(X_{n}\right)_{n \in \mathbb{N}}$ converges pointwise to the variable $Y: \Omega \longrightarrow \mathbb{R}$ defined as follows: for $t \in \Omega$

$$
Y(t)= \begin{cases}\sum_{i=1}^{m} \frac{c_{i}}{k^{\alpha_{i}} P\left(A_{k}\right)}, & \text { when } t \in A_{k}, \text { for } k \in \mathbb{N} \\ \sum_{i=1}^{m} \frac{-c_{i}}{k^{\alpha_{i} P}\left(B_{k}\right)}, & \text { when } t \in B_{k}, \text { for } k \in \mathbb{N} .\end{cases}
$$

On the other hand, we have the following estimation for every $n \in \mathbb{N}$ :

$$
\begin{aligned}
& E\left|X_{n}\right| \\
& \quad \geq E\left(\left|c_{1}\right|\left|X_{n}^{\left(\alpha_{1}\right)}\right|-\left|c_{2}\right|\left|X_{n}^{\left(\alpha_{2}\right)}\right|-\cdots-\left|c_{m}\right|\left|X_{n}^{\left(\alpha_{m}\right)}\right|\right) \\
& \quad=\sum_{k=1}^{n}\left(\frac{2\left|c_{1}\right|}{k^{\alpha_{1}}}-\frac{2\left|c_{2}\right|}{k^{\alpha_{2}}}-\cdots-\frac{2\left|c_{m}\right|}{k^{\alpha_{m}}}\right) \\
& =\sum_{k=1}^{n}\left(\frac{2\left|c_{1}\right|}{k^{\alpha_{1}}}\left(1-\frac{\left|c_{2}\right|}{\left|c_{1}\right| k^{\alpha_{2}-\alpha_{1}}}-\cdots-\frac{\left|c_{m}\right|}{\left|c_{1}\right| k^{\alpha_{m}-\alpha_{1}}}\right)\right) .
\end{aligned}
$$

Since $\alpha_{1}<\alpha_{2}<\cdots<\alpha_{m}$, there is $K \in \mathbb{N}$ such that for every $k \geq K$

$$
1-\frac{\left|c_{2}\right|}{\left|c_{1}\right| k^{\alpha_{2}-\alpha_{1}}}-\cdots-\frac{\left|c_{m}\right|}{\left|c_{1}\right| k^{\alpha_{m}-\alpha_{1}}} \geq \frac{1}{2} .
$$

In particular, for every $n \geq K$ we have

$$
\begin{aligned}
& \sum_{k=K}^{n}\left(\frac{2\left|c_{1}\right|}{k^{\alpha_{1}}}\left(1-\frac{\left|c_{2}\right|}{\left|c_{1}\right| k^{\alpha_{2}-\alpha_{1}}}-\cdots-\frac{\left|c_{m}\right|}{\left|c_{1}\right| k^{\alpha_{m}-\alpha_{1}}}\right)\right) \\
& \quad \geq \sum_{k=K}^{n} \frac{\left|c_{1}\right|}{k^{\alpha_{1}}} \stackrel{n \rightarrow \infty}{\longrightarrow} \infty .
\end{aligned}
$$

Hence,

$$
E\left|X_{n}\right| \longrightarrow \infty
$$

as required. 
In the martingales setting one may find another somehow surprising example, namely a martingale such that it converges to 0 in measure but not almost surely (see [9]). The next theorem is based on this construction but provides a large structure of such martingales.

Theorem 11. Let $(\Omega, \mathcal{S}, P)=\left([0,1]^{\mathbb{N}}, \mathscr{B} \operatorname{or}\left([0,1]^{\mathbb{N}}\right), P\right)$ be a probability space, where $\mathscr{B}$ or $\left([0,1]^{\mathbb{N}}\right)$ is the $\sigma$-algebra of Borel subsets of $[0,1]^{\mathbb{N}}$ and $P: \mathscr{B o r}\left([0,1]^{\mathbb{N}}\right) \rightarrow[0,1]$ is the product measure (countable product of the Lebesgue measure on $[0,1])$. There exist a filtration $\left(\mathscr{F}_{n}\right)_{n \in \mathbb{N}}$ and a $\mathbf{c}$-dimensional vector space $\mathbb{V}$ of martingales adapted to the filtration $\left(\mathscr{F}_{n}\right)_{n \in \mathbb{N}}$ such that every nonzero $\left(X_{n}\right)_{n \in \mathbb{N}} \in \mathbb{V}$ converges to 0 in measure but no almost surely.

Proof. For $\alpha \in(1 / 3,1 / 2]$ and $n \in \mathbb{N}$ define a random variable $Z_{n}^{(\alpha)}:[0,1]^{\mathbb{N}} \longrightarrow \mathbb{R}$ by the formula:

$$
Z_{n}^{(\alpha)}\left(\left(\omega_{k}\right)_{k \in \mathbb{N}}\right)= \begin{cases}1, & \text { if } \omega_{n}<\frac{1}{2 n^{\alpha}} \\ -1, & \text { if } \omega_{n}>1-\frac{1}{2 n^{\alpha}} \\ 0, & \text { elsewhere, }\end{cases}
$$

for $\left(\omega_{k}\right)_{k \in \mathbb{N}} \in[0,1]^{\mathbb{N}}$. Clearly, for any $\alpha \in(1 / 3,1 / 2]$ and any natural numbers $i \neq j$ the random variables $Z_{i}^{(\alpha)}$ and $Z_{j}^{(\alpha)}$ are independent. way:

Now, let us define a sequence $\left(X_{n}^{(\alpha)}\right)_{n \in \mathbb{N}}$ in the following

$$
\begin{aligned}
& X_{1}^{(\alpha)}=Z_{1}^{(\alpha)} \\
& X_{n}^{(\alpha)}=Z_{n}^{(\alpha)} \mathbb{1}_{\left\{X_{n-1}^{(\alpha)}=0\right\}}+n^{\alpha} X_{n-1}^{(\alpha)}\left|Z_{n}^{(\alpha)}\right| \mathbb{1}_{\left\{X_{n-1}^{(\alpha)} \neq 0\right\}},
\end{aligned}
$$

for $n \geq 2$.

We will show that $\left(X_{n}^{(\alpha)}\right)_{n \in \mathbb{N}}$ forms a martingale adapted to the filtration $\left(\mathscr{F}_{n}\right)_{n \in \mathbb{N}}$, where

$$
\mathscr{F}_{n}=\left\{A \times[0,1]^{\mathbb{N} \backslash\{1, \ldots, n\}}: A \in \mathscr{B} \text { or }\left([0,1]^{n}\right)\right\},
$$

for $n \in \mathbb{N}$.

For every $n \in \mathbb{N}$, we have that

(1) $X_{n}^{(\alpha)}$ is $\mathscr{F}_{n}$ measurable by the definition,

(2) $E\left|X_{n}^{(\alpha)}\right| \leq(n !)^{\alpha}<\infty$ as $E\left|X_{1}^{(\alpha)}\right|=1$ and $\left|X_{n}^{(\alpha)}\right| \leq$ $n^{\alpha}\left|X_{n-1}^{(\alpha)}\right|$, for every $n>1$,

(3) by Remark 5, we have the following equalities (almost sure):

$$
\begin{aligned}
& E\left(X_{n+1}^{(\alpha)} \mid \mathscr{F}_{n}\right) \\
&= E\left(Z_{n+1}^{(\alpha)} \mathbb{1}_{\left\{X_{n}^{(\alpha)}=0\right\}} \mid \mathscr{F}_{n}\right) \\
&+E\left((n+1)^{\alpha} X_{n}^{(\alpha)}\left|Z_{n+1}^{(\alpha)}\right| \mathbb{1}_{\left\{X_{n}^{(\alpha)} \neq 0\right\}} \mid \mathscr{F}_{n}\right) \\
&= \mathbb{1}_{\left\{X_{n}^{(\alpha)}=0\right\}} E\left(Z_{n+1}^{(\alpha)} \mid \mathscr{F}_{n}\right) \\
&+(n+1)^{\alpha} X_{n}^{(\alpha)} \mathbb{1}_{\left\{X_{n}^{(\alpha)} \neq 0\right\}} E\left(\left|Z_{n+1}^{(\alpha)}\right| \mid \mathscr{F}_{n}\right) \\
&= \mathbb{1}_{\left\{X_{n}^{(\alpha)}=0\right\}} E\left(Z_{n+1}^{(\alpha)}\right)
\end{aligned}
$$

$$
\begin{aligned}
& +(n+1)^{\alpha} X_{n}^{(\alpha)} \mathbb{1}_{\left\{X_{n}^{(\alpha)} \neq 0\right\}} E\left(\left|Z_{n+1}^{(\alpha)}\right|\right) \\
= & 0+(n+1)^{\alpha} X_{n}^{(\alpha)} \mathbb{1}_{\left\{X_{n}^{(\alpha)} \neq 0\right\}} \frac{1}{(n+1)^{\alpha}}=X_{n}^{(\alpha)} .
\end{aligned}
$$

Let $\mathbb{V}=\operatorname{span}\left\{\left(X_{n}^{(\alpha)}\right)_{n \in \mathbb{N}}: \alpha \in(1 / 3,1 / 2]\right\}$, which is a vector space of martingales adapted to the filtration $\left(\mathscr{F}_{n}\right)_{n \in \mathbb{N}}$. We will show that $\mathbb{V}$ has the desired properties. Let $X=\left(X_{n}\right)_{n \in \mathbb{N}} \in \mathbb{V}$ be a nonzero martingale; i.e., there are $m \in \mathbb{N}, c_{1}, \ldots, c_{m} \in$ $\mathbb{R} \backslash\{0\}$ and $1 / 3<\alpha_{m}<\cdots<\alpha_{2}<\alpha_{1} \leq 1 / 2$ such that $X=\sum_{i=1}^{m} c_{i} X^{\left(\alpha_{i}\right)}$; that is, $X_{n}=\sum_{i=1}^{m} c_{i} X_{n}^{\left(\alpha_{i}\right)}$ for every $n \in \mathbb{N}$. Observe that, by the definition of $X_{n}^{(\alpha)}$, we have that for every $t \in \Omega$

$$
X_{n}^{(\alpha)}(t)=0 \Longleftrightarrow Z_{n}^{(\alpha)}(t)=0,
$$

hence

$$
\begin{aligned}
Z_{n}^{\left(\alpha_{m}\right)}(t) & =0 \Longrightarrow \\
\forall_{i \leq m} Z_{n}^{\left(\alpha_{i}\right)}(t) & =0 \Longrightarrow \\
\forall_{i \leq m} X_{n}^{\left(\alpha_{i}\right)}(t) & =0 \Longrightarrow \\
X_{n}(t) & =0 .
\end{aligned}
$$

Therefore

$$
P\left(X_{n}=0\right) \geq P\left(Z_{n}^{\left(\alpha_{m}\right)}=0\right)=1-\frac{1}{n^{\alpha_{m}}}
$$

and in particular $P\left(X_{n}=0\right) \longrightarrow 1$ with $n \longrightarrow \infty$; i.e., $\left(X_{n}\right)_{n \in \mathbb{N}}$ converges to 0 in measure. To prove the second assertion, let us firstly observe the following.

Claim 1. For every $\alpha \in(1 / 3,1 / 2]$ and any $n \in \mathbb{N}$ with $n \geq 3$, we have

$$
P\left(\left|X_{n}^{(\alpha)}\right| \leq n^{\alpha}\right)=1-\frac{1}{(n(n-1)(n-2))^{\alpha}}
$$

and the event $\left|X_{n}^{(\alpha)}\right| \leq n^{\alpha}$ depends, at most, on the events $\left|Z_{i}^{(\alpha)}\right|=j$ for $i \in\{n, n-1, n-2\}$ and $j \in\{0,1\}$.

Proof. By an easy inductive argument we may prove that

$$
X_{n}^{(\alpha)}[\Omega] \subseteq\left\{0, \pm 1, \pm n^{\alpha}, \pm(n(n-1))^{\alpha}, \ldots, \pm(n !)^{\alpha}\right\},
$$

so for $t \in \Omega$,

$$
\begin{aligned}
\left|X_{n}^{(\alpha)}(t)\right| & \leq n^{\alpha} \Longleftrightarrow X_{n}^{(\alpha)}(t)=0 \vee\left|X_{n}^{(\alpha)}(t)\right| \\
& =1 \vee\left|X_{n}^{(\alpha)}(t)\right|=n^{\alpha} .
\end{aligned}
$$

By the definition, we have the following: for $t \in \Omega$,

$$
\begin{aligned}
X_{n}^{(\alpha)}(t) & =0 \Longleftrightarrow Z_{n}^{(\alpha)}(t)=0, \\
X_{n}^{(\alpha)}(t) & =1 \Longleftrightarrow\left|Z_{n}^{(\alpha)}(t)\right|=1 \wedge X_{n-1}^{(\alpha)}(t)=0 \Longleftrightarrow \\
\left|Z_{n}^{(\alpha)}(t)\right| & =1 \wedge Z_{n-1}^{(\alpha)}(t)=0, \\
X_{n}^{(\alpha)}(t) & =n^{\alpha} \Longleftrightarrow\left|Z_{n}^{(\alpha)}(t)\right|=1 \wedge\left|X_{n-1}^{(\alpha)}(t)\right|=1 \Longleftrightarrow \\
\left|Z_{n}^{(\alpha)}(t)\right| & =1 \wedge\left|Z_{n-1}^{(\alpha)}(t)\right|=1 \wedge Z_{n-2}^{(\alpha)}(t)=0 .
\end{aligned}
$$


Clearly the above events are independent so

$$
\begin{aligned}
P\left(\left|X_{n}^{(\alpha)}\right| \leq n^{\alpha}\right) & \\
= & P\left(Z_{n}^{(\alpha)}=0\right)+P\left(\left|Z_{n}^{(\alpha)}\right|=1 \wedge Z_{n-1}^{(\alpha)}=0\right) \\
& +P\left(\left|Z_{n}^{(\alpha)}\right|=1 \wedge\left|Z_{n-1}^{(\alpha)}\right|=1 \wedge Z_{n-2}^{(\alpha)}=0\right) \\
= & 1-\frac{1}{n^{\alpha}}+\frac{1}{n^{\alpha}}\left(1-\frac{1}{(n-1)^{\alpha}}\right) \\
& +\frac{1}{n^{\alpha}} \frac{1}{(n-1)^{\alpha}}\left(1-\frac{1}{(n-2)^{\alpha}}\right) \\
= & 1-\frac{1}{(n(n-1)(n-2))^{\alpha} .}
\end{aligned}
$$

The second assertion is a direct consequence of the proof.

Let $A_{n}=\left\{t \in \Omega:\left|X_{n}^{\left(\alpha_{1}\right)}(t)\right| \geq n^{\alpha_{1}} \wedge \forall_{i \leq m}\left|X_{n}^{\left(\alpha_{i}\right)}(t)\right| \leq n^{\alpha_{i}}\right\}$ for $n \in \mathbb{N}$. By the Claim, we have the following estimation:

$$
\begin{aligned}
P\left(A_{n}\right) \geq & P\left(\left|X_{n}^{\left(\alpha_{1}\right)}\right| \geq n^{\alpha_{1}}\right)-\sum_{i \leq m} \frac{1}{(n(n-1)(n-2))^{\alpha_{i}}} \\
= & P\left(\left|Z_{n}^{\left(\alpha_{1}\right)}\right|=1 \wedge X_{n-1}^{\left(\alpha_{1}\right)} \neq 0\right) \\
& -\sum_{i \leq m} \frac{1}{(n(n-1)(n-2))^{\alpha_{i}}} \\
= & P\left(\left|Z_{n}^{\left(\alpha_{1}\right)}\right|=1 \wedge\left|Z_{n-1}^{\left(\alpha_{1}\right)}\right|=1\right) \\
& -\sum_{i \leq m} \frac{1}{(n(n-1)(n-2))^{\alpha_{i}}} \\
= & \frac{1}{n^{\alpha_{1}}} \frac{1}{(n-1)^{\alpha_{1}}}-\sum_{i \leq m} \frac{1}{(n(n-1)(n-2))^{\alpha_{i}}}
\end{aligned}
$$

and $\sum_{n=1}^{\infty} P\left(A_{3 n}\right)=\infty\left(\right.$ as $\alpha_{i} \in(1 / 3,1 / 2]$ for $\left.i \leq m\right)$. Moreover, by the Claim, the events $A_{3}, A_{6}, A_{9}, \ldots$ are independent so by the Borel-Cantelli Lemma

$$
P\left(\limsup _{n \rightarrow \infty} A_{3 n}\right)=1 \text {. }
$$

Since $\alpha_{m}<\cdots<\alpha_{2}<\alpha_{1}$, there exists $M \in \mathbb{N}$ such that for all $n \geq M$ we have

$$
\left|c_{1}\right| n^{\alpha_{1}}-\sum_{i=2}^{m}\left|c_{i}\right| n^{\alpha_{i}}>\frac{\left|c_{1}\right| n^{\alpha_{1}}}{2}>1 .
$$

Let $n \geq M$ and $t \in A_{n}$. Then

$$
\begin{aligned}
\left|X_{n}(t)\right| & \geq\left|c_{1}\right|\left|X_{n}^{\left(\alpha_{1}\right)}(t)\right|-\sum_{i=2}^{m}\left|c_{i}\right|\left|X_{n}^{\left(\alpha_{i}\right)}(t)\right| \\
& \stackrel{t \in A_{n}}{\geq}\left|c_{1}\right| n^{\alpha_{1}}-\sum_{i=2}^{m}\left|c_{i}\right| n^{\alpha_{i}}>1 .
\end{aligned}
$$

In particular, by $(\star)$,

$$
P\left(X_{n} \neq 0 \text { for infinitely many } n\right)=1 ;
$$

hence $\left(X_{n}\right)_{n \in \mathbb{N}}$ does not tend to 0 almost surely. In particular, $\mathbb{V}$ is $\mathbf{c}$-dimensional vector space.

(End of Proof)

\section{Kolmogorov's Theorem}

We start this section with recalling a well-known theorem due to Kolmogorov (see [7]).

Theorem 12 (Kolmogorov's theorem, [7]). Let $(\Omega, \mathcal{S}, P)$ be a probability space and $\left(X_{n}\right)_{n \in \mathbb{N}}$ be a sequence of independent random variables such that $E X_{n}=0$ for all $n \in \mathbb{N}$ and $\sum_{n=1}^{\infty} \operatorname{var} X_{n}<\infty$. Then, for $S_{n}=X_{1}+\cdots+X_{n}$ the sequence $\left(S_{n}\right)_{n \in \mathbb{N}}$ is convergent almost surely.

Examples of sequences of independent random variables with expected values equal to 0 and divergent series of their variations such that $\left(X_{1}+\cdots+X_{n}\right)_{n \in \mathbb{N}}$ does converge almost surely are known (see [9]). Here we show that there is a $\mathfrak{c}$ dimensional vector space of such sequences.

Theorem 13. There exist a probability space $(\Omega, \mathcal{S}, P)$ and a $\mathrm{c}$ dimensional vector subspace $\mathbb{V} \subseteq\left(L_{2}(\Omega, \mathcal{S}, P)\right)^{\mathbb{N}}$ of sequences of independent random variables, such that for every nonzero $X=\left(X_{n}\right)_{n \in \mathbb{N}} \in \mathbb{V}$ we have $E X_{n}=0$ for every $n \in \mathbb{N}$, $\sum_{n=1}^{\infty} \operatorname{var} X_{n}=\infty$, and $\left(X_{1}+\cdots+X_{n}\right)_{n \in \mathbb{N}}$ converges almost surely.

Proof. Let $(\Omega, \mathcal{S}, P)$ be a probability space such that there exists a sequence of independent random variables $\left(Z_{n}\right)_{n \in \mathbb{N}}$ such that

$$
\begin{aligned}
& P\left(Z_{n}=n\right)=P\left(Z_{n}=-n\right)=\frac{1}{2 n^{2}} \text { and } \\
& P\left(Z_{n}=0\right)=1-\frac{1}{n^{2}} .
\end{aligned}
$$

Clearly, $E Z_{n}=0$ and $\operatorname{var} Z_{n}=1$ for any $n \in \mathbb{N}$, so $\sum_{n=1}^{\infty} \operatorname{var} X_{n}=\infty$. Let $\left\{I_{\alpha}: \alpha<\mathfrak{c}\right\}$ be an almost disjoint (i.e., an intersection of any two different members is finite) family of infinite subsets of $\mathbb{N}$. For $\alpha<\mathfrak{c}$ and $n \in \mathbb{N}$, define a random variable $X_{n}^{(\alpha)}$ by the formula:

$$
X_{n}^{(\alpha)}= \begin{cases}Z_{n}, & \text { if } n \in I_{\alpha} \\ 0, & \text { if } n \notin I_{\alpha}\end{cases}
$$

and observe that $E X_{n}^{(\alpha)}=0$ and var $X_{n}^{(\alpha)} \leq 1$. Hence, $X_{n}^{(\alpha)} \epsilon$ $L_{2}(\Omega, \mathcal{S}, P)$ for any $\alpha<\mathfrak{c}$ and $n \in \mathbb{N}$. Let $\mathbb{V}$ be the subspace of $\left(L_{2}(\Omega, \mathcal{S}, P)\right)^{\mathbb{N}}$ spanned by $\left\{\left(X_{n}^{(\alpha)}\right)_{n \in \mathbb{N}}: \alpha<\mathfrak{c}\right\}$. We will show that $\mathbb{V}$ has the desired properties. Let $X=\left(X_{n}\right)_{n \in \mathbb{N}} \in$ $\mathbb{V}$ be of the form $\left(X_{n}\right)_{n \in \mathbb{N}}=\left(\sum_{i=1}^{m} c_{i} X_{n}^{\left(\alpha_{i}\right)}\right)_{n \in \mathbb{N}}$, for some $m \in$ $\mathbb{N}, c_{1}, \ldots, c_{m} \in \mathbb{R} \backslash\{0\}$, and $\alpha_{1}<\cdots<\alpha_{m}<$ c. Clearly, $\left(X_{n}\right)_{n \in \mathbb{N}}$ is a sequence of independent random variables (by the independence of $\left.\left(Z_{n}\right)_{n \in \mathbb{N}}\right)$ and $E X_{n}=0$ for all $n \in \mathbb{N}$. Moreover, for infinitely many naturals, namely, from the set $I_{\alpha_{1}} \backslash \bigcup_{i=2}^{m}\left(I_{\alpha_{1}} \cap I_{\alpha_{i}}\right)$, we have var $X_{n}=\left|c_{1}\right| \operatorname{var} Z_{n}=\left|c_{1}\right|$ so $\sum_{n=1}^{\infty} \operatorname{var} X_{n}=\infty$. In particular, $\mathbb{V}$ is $\mathfrak{c}$-dimensional vector space. As $\left\{I_{\alpha}: \alpha<\mathfrak{c}\right\}$ is almost disjoint, there is $M \in \mathbb{N}$ 
such that the sets $I_{\alpha_{i}} \backslash\{1, \ldots, M\}(i \in\{1, \ldots, m\})$ are pairwise disjoint. Let $n>M$ and observe that

$$
P\left(X_{n} \neq 0\right) \leq P\left(Z_{n} \neq 0\right)=\frac{1}{n^{2}}
$$

therefore $\sum_{n=1}^{\infty} P\left(X_{n} \neq 0\right)<\infty$. By the Borel-Cantelli Lemma

$$
P\left(X_{n} \neq 0 \text { for infinitely many } n\right)=0
$$

so

$$
P\left(X_{n}=0 \text { for almost all } n\right)=1 .
$$

In particular, $\left(X_{1}+\cdots+X_{n}\right)_{n \in \mathbb{N}}$ converges almost surely.

\section{Failure of Fubini's Theorem}

The famous Theorem of Fubini states that for any integrable function $f:[0,1] \times[0,1] \longrightarrow \mathbb{R}$ the following equality holds:

$$
\begin{aligned}
& \int_{[0,1]}\left(\int_{[0,1]} f(x, y) d x\right) d y \\
& \quad=\int_{[0,1]}\left(\int_{[0,1]} f(x, y) d y\right) d x
\end{aligned}
$$

(from now on integrable, measurable, almost every (a.e.) means Lebesgue integrable, Lebesgue measurable, from a conull set). The idea of looking for examples of functions $f$ : $[0,1] \times[0,1] \longrightarrow \mathbb{R}$ (measurable unbounded or nonmeasurable) attracted an attention of many mathematicians (see, for example, [10-13]). In this section, we will present some lineability results (both positive and negative) in this area.

5.1. The Case of Bounded Functions. Let $f:[0,1] \times[0,1] \longrightarrow$ $\mathbb{R}$ be a bounded function. We say that for $f$ the Iterated Integral Property Failure holds when

(IPPF)

(i) $[0,1] \ni x \longmapsto f(x, y)$ is integrable for almost every $y \in[0,1]$,

$[0,1] \ni y \longmapsto f(x, y)$ is integrable for almost every $x \in[0,1]$,

(ii) $[0,1] \ni x \stackrel{\varphi_{f}}{\longmapsto} \int_{[0,1]} f(x, y) d y$, defined a.e., is integrable,

$[0,1] \ni y \stackrel{\psi_{f}}{\longmapsto} \int_{[0,1]} f(x, y) d x$, defined a.e., is integrable,

(iii) $\int_{[0,1]} \varphi_{f}(x) d x \neq \int_{[0,1]} \psi_{f}(y) d y$.

It is known that existence of a bounded function $f:[0,1] \times$ $[0,1] \longrightarrow \mathbb{R}$ with (IIPF) is independent from $Z F C$ (we refer the reader to [14] for the details). In this situation, we may obtain some results consistently with ZFC.

Theorem 14. It is consistent with ZFC that the set of bounded functions $f:[0,1] \times[0,1] \longrightarrow \mathbb{R}$ with (IIPF) is 1-lineable (or star-like) but not 2-lineable.
Proof. Let $S \subseteq[0,1] \times[0,1]$ be the so-called Steinhaus set, i.e., a set with all horizontal sections of measure zero and all vertical sections of full measure (existence of such a set is consistent with ZFC; for example, it can be constructed under the Continuum Hypothesis; see [14]). It is easy to see that, for the characteristic function $f_{S}:[0,1] \times[0,1] \longrightarrow \mathbb{R}$ of $S$ and any $a \in \mathbb{R} \backslash\{0\}$, the function $a f_{S}$ has (IIPF).

To prove the second assertion, let $f, g:[0,1] \times[0,1] \longrightarrow$ $\mathbb{R}$ be bounded and satisfy (IIPF). Denote (the real numbers) $A_{f}=\int_{[0,1]} \varphi_{f}(x) d x, B_{f}=\int_{[0,1]} \psi_{f}(y) d y$ and $A_{g}, B_{g}$ in analogous way. Then $A_{f} \neq B_{f} A_{g} \neq B_{g}$. It is easy to see that for nonzero numbers $a=\left(A_{g}-B_{g}\right) /\left(A_{f}-B_{f}\right), b=-1$, and the function $h=a f+b g$, we have

$$
\begin{aligned}
& \int_{[0,1]} \varphi_{h}(x) d x-\int_{[0,1]} \psi_{h}(y) d y \\
& \quad=\frac{A_{g}-B_{g}}{A_{f}-B_{f}} A_{f}-A_{g}-\frac{A_{g}-B_{g}}{A_{f}-B_{f}} B_{f}+B_{g}=0
\end{aligned}
$$

so $h$ does not have (IIPF) and the proof is finished.

Remark 15. A modification of a function with (IIPF) on the set of measure zero still has (IIPF). Hence, there are $2^{\mathfrak{c}}$ distinct bounded functions with (IIPF).

5.2. The Case of Unbounded Functions. It is natural to ask a question about the (IIPF) condition for an unbounded function $f:[0,1] \times[0,1] \longrightarrow \mathbb{R}$ (an example of such function exists in $Z F C$; see [14]). Let us start with an easy observation, based on an analogous argument as in Theorem 14.

Theorem 16. The set of unbounded functions $f:[0,1] \times$ $[0,1] \longrightarrow \mathbb{R}$ with (IIPF) is 1-lineable (or star-like) but not 2lineable.

Proof. Let $f:[0,1] \times[0,1] \longrightarrow \mathbb{R}$ be an unbounded function with (IIPF) (see [14]). It is easy to check that for any $a \in \mathbb{R} \backslash\{0\}$ we have that of has (IIPF), what proves 1-lineability. The second assertion is a consequence of a property (ii) in (IIPF), where we actually assume that the integrals $\int_{[0,1]} \varphi_{f}(x) d x$, $\int_{[0,1]} \psi_{f}(y) d y$ (even for an unbounded $f$ ) are finite and the same argument as in the proof of Theorem 14 works.

Having in mind the above result, in the context of an unbounded function $f:[0,1] \times[0,1] \longrightarrow \mathbb{R}$, it is natural to reformulate the (IIPF) condition as follows:

$\left(\mathrm{IPPF}_{\infty}\right)$

(i) $[0,1] \ni x \longmapsto f(x, y)$ is integrable for almost every $y \in[0,1]$,

$[0,1] \ni y \longmapsto f(x, y)$ is integrable for almost every $x \in[0,1]$,

(ii) $[0,1] \ni x \stackrel{\varphi_{f}}{\longmapsto} \int_{[0,1]} f(x, y) d y$, defined a.e., is measurable and the integral $\int_{[0,1]} \varphi_{f}(x) d x$ exists (is finite or infinite), 
$[0,1] \ni y \stackrel{\psi_{f}}{\longmapsto} \int_{[0,1]} f(x, y) d x$, defined a.e., is measurable and the integral $\int_{[0,1]} \psi_{f}(y) d y$ exists (is finite or infinite),

(iii) $\int_{[0,1]} \varphi_{f}(x) d x \neq \int_{[0,1]} \psi_{f}(y) d y$.

Theorem 17. The set of unbounded functions $f:[0,1] \times$ $[0,1] \longrightarrow \mathbb{R}$ with $\left(I I P F_{\infty}\right)$ is c-lineable.

Proof. Let $\alpha \in(2, \infty)$ and let $a_{n}=1 / 2^{n-1}$ for $n \in \mathbb{N}$. Define a function $f^{(\alpha)}:[0,1] \times[0,1] \longrightarrow \mathbb{R}$ in the following way: for any $n \in \mathbb{N}$ and any $x \in\left(a_{n+1}, a_{n}\right]$

$$
f^{(\alpha)}(x, y)= \begin{cases}-\alpha^{2 n}, & \text { when } y \in\left[0, a_{n+1}\right) \\ \alpha^{2 n}, & \text { when } y \in\left[a_{n+1}, a_{n}\right) \\ 0, & \text { when } y \in\left[a_{n}, 1\right]\end{cases}
$$

and $f^{(\alpha)}(0, y)=0$ for $y \in[0,1]$. Let $\alpha>2$. Observe that $\varphi_{f^{(\alpha)}}(x)=0$, for any $x \in[0,1]$. On the other hand, for any $n \in \mathbb{N}$ and any $y \in\left[a_{n+1}, a_{n}\right)$, we have

$$
\begin{aligned}
\psi_{f^{(\alpha)}}(y) & =\frac{\alpha^{2 n}}{2^{n}}-\sum_{k=1}^{n-1} \frac{\alpha^{2 k}}{2^{k}}=A_{\alpha}^{n}-A_{\alpha} \frac{1-A_{\alpha}^{n-1}}{1-A_{\alpha}} \\
& =\frac{A_{\alpha}^{n+1}-2 A_{\alpha}^{n}+A_{\alpha}}{A_{\alpha}-1}>0,
\end{aligned}
$$

where $A_{\alpha}=\alpha^{2} / 2$. For the simplicity, let us denote $B_{\alpha}^{(n)}=$ $\left(A_{\alpha}^{n+1}-2 A_{\alpha}^{n}+A_{\alpha}\right) /\left(A_{\alpha}-1\right)$.

Let $\mathbb{V}=\operatorname{span}\left\{f^{(\alpha)}: \alpha \in(2, \infty)\right\}$; we will show that every nonzero element of $\mathbb{V}$ has (IIPF $\left.{ }_{\infty}\right)$. Let $f \in \mathbb{V}$ be of the form $f=\sum_{i=1}^{m} c_{i} f^{\left(\alpha_{i}\right)}$ for some $m \in \mathbb{N}, c_{1}, \ldots, c_{m} \in \mathbb{R} \backslash\{0\}$ and $\alpha_{1}>\cdots>\alpha_{m}>2$. Clearly, for all $x \in[0,1]$, we have that $\varphi_{f}(x)=0$ as $\varphi_{f^{\left(\alpha_{i}\right)}}(x)=0$ for every $i \in\{1, \ldots, m\}$. Therefore, $\int_{[0,1]} \varphi_{f}(x) d x=0$. On the other hand, we have

$$
\begin{aligned}
\int_{[0,1]} \psi_{f}(y) d y & =\sum_{n=1}^{\infty} \frac{1}{2^{n}}\left(\sum_{i=1}^{m} c_{i} B_{\alpha_{i}}^{(n)}\right) \\
& =\sum_{n=1}^{\infty}\left(\sum_{i=1}^{m} c_{i} \frac{B_{\alpha_{i}}^{(n)}}{2^{n}}\right) .
\end{aligned}
$$

Observe that, as $\alpha_{1}>\alpha_{2}>\cdots>\alpha_{m}$,

$$
\frac{B_{\alpha_{i}}^{(n)}}{B_{\alpha_{1}}^{(n)}} \stackrel{n \rightarrow \infty}{\longrightarrow} 0,
$$

for any $i \in\{2, \ldots, m\}$. Hence, there is $M \in \mathbb{N}$ such that for any $n \geq M$

$$
-\frac{1}{2}<\frac{2 c_{2} B_{\alpha_{2}}^{(n)}}{c_{1} B_{\alpha_{1}}^{(n)}}+\cdots+\frac{2 c_{m} B_{\alpha_{m}}^{(n)}}{c_{1} B_{\alpha_{1}}^{(n)}}<\frac{1}{2} .
$$

Let $n \geq M$. We have two possibilities:
(1) If $c_{1}>0$, then $\sum_{i=1}^{m} c_{i}\left(B_{\alpha_{i}}^{(n)} / 2^{n}\right)>c_{1} B_{\alpha_{1}}^{(n)} / 2^{n+1}$. Indeed,

$$
\begin{gathered}
\sum_{i=1}^{m} c_{i} \frac{B_{\alpha_{i}}^{(n)}}{2^{n}}-\frac{c_{1} B_{\alpha_{1}}^{(n)}}{2^{n+1}}=\frac{c_{1} B_{\alpha_{1}}^{(n)}}{2^{n+1}}+\frac{c_{2} B_{\alpha_{2}}^{(n)}}{2^{n}}+\cdots+\frac{c_{m} B_{\alpha_{m}}^{(n)}}{2^{n}} \\
=\frac{c_{1} B_{\alpha_{1}}^{(n)}}{2^{n+1}}\left(1+\frac{2 c_{2} B_{\alpha_{2}}^{(n)}}{c_{1} B_{\alpha_{1}}^{(n)}}+\cdots+\frac{2 c_{m} B_{\alpha_{m}}^{(n)}}{c_{1} B_{\alpha_{1}}^{(n)}}\right)>0 .
\end{gathered}
$$

(2) If $c_{1}<0$, then $\sum_{i=1}^{m} c_{i}\left(B_{\alpha_{i}}^{(n)} / 2^{n}\right)<c_{1} B_{\alpha_{1}}^{(n)} / 2^{n+1}$. Indeed,

$$
\begin{gathered}
\sum_{i=1}^{m} c_{i} \frac{B_{\alpha_{i}}^{(n)}}{2^{n}}-\frac{c_{1} B_{\alpha_{1}}^{(n)}}{2^{n+1}}=\frac{c_{1} B_{\alpha_{1}}^{(n)}}{2^{n+1}}+\frac{c_{2} B_{\alpha_{2}}^{(n)}}{2^{n}}+\cdots+\frac{c_{m} B_{\alpha_{m}}^{(n)}}{2^{n}} \\
=\frac{c_{1} B_{\alpha_{1}}^{(n)}}{2^{n+1}}\left(1+\frac{2 c_{2} B_{\alpha_{2}}^{(n)}}{c_{1} B_{\alpha_{1}}^{(n)}}+\cdots+\frac{2 c_{m} B_{\alpha_{m}}^{(n)}}{c_{1} B_{\alpha_{1}}^{(n)}}\right)<0 .
\end{gathered}
$$

Moreover, $B_{\alpha_{1}}^{(n)} / 2^{n+1} \stackrel{n \longrightarrow \infty}{\longrightarrow} \infty$, because $\alpha_{1}>2$. In particular,

$$
\int_{[0,1]} \psi_{f}(y) d y= \begin{cases}\infty, & \text { when } c_{1}>0 \\ -\infty, & \text { when } c_{1}<0 .\end{cases}
$$

The above implies that $f$ satisfies $\left(\operatorname{IIPF}_{\infty}\right)$ and that $\mathbb{V}$ is $\mathfrak{c}$ dimensional.

Finally, we show that the last theorem is optimal in the sense of cardinalities.

Theorem 18. The set of unbounded functions $f:[0,1] \times$ $[0,1] \longrightarrow \mathbb{R}$ with $\left(I I P F_{\infty}\right)$ is not $\mathrm{c}^{+}$-lineable.

Proof. Suppose on the contrary that the set is $\mathrm{c}^{+}$-lineable and let $f^{\xi}::[0,1] \times[0,1] \longrightarrow \mathbb{R}$, for $\xi<\mathfrak{c}^{+}$, have $\left(\operatorname{IIPF}_{\infty}\right)$ and be such that for any $m \in \mathbb{N}, c_{1}, \ldots, c_{m} \in \mathbb{R} \backslash\{0\}$ and any $\xi_{1}<\cdots<\xi_{m}<\boldsymbol{c}^{+}$the function $f=\sum_{i=1}^{m} c_{i} f^{\xi_{i}}$ has $\left(\mathrm{IIPF}_{\infty}\right)$. By the assumption, for any $\xi<\mathfrak{c}^{+}$, there exist (defined a.e.) measurable functions $\varphi_{f^{\xi}}, \psi_{f^{\xi}}$. It is known that any measurable function is equal to a Borel function almost everywhere; i.e., there exist Borel functions $\bar{\varphi}_{f^{\xi}}, \bar{\psi}_{f^{\xi}}$ such that $\bar{\varphi}_{f^{\xi}}=\varphi_{f}$ almost everywhere and $\bar{\psi}_{f^{\xi}}=\psi_{f^{\xi}}$ almost everywhere. As there are $c$ many distinct Borel functions, there exist $\xi<\zeta<\mathfrak{c}^{+}$such that $\bar{\varphi}_{f^{\xi}}=\bar{\varphi}_{f^{\zeta}}, \bar{\psi}_{f^{\xi}}=\bar{\psi}_{f^{\zeta}}$. Clearly, for the function $f=f^{\xi}-f^{\zeta}$ we have $\varphi_{f}=\varphi_{f^{\xi}}-\varphi_{f^{\zeta}}=\bar{\varphi}_{f^{\xi}}-$ $\bar{\varphi}_{f^{\zeta}}=0$ almost everywhere and $\int_{[0,1]} \varphi_{f}(x) d x=0$. Similarly, $\int_{[0,1]} \psi_{f}(y) d y=0$ and we reach a contradiction.

\section{Data Availability}

No data were used to support this study.

\section{Conflicts of Interest}

The authors declare that they have no conflicts of interest. 


\section{Acknowledgments}

The results from the Section Peano Curves and Semigroupability were a part of a very first version of the paper [1]. We would like to thank Professor T. Natkaniec for the agreement to include these outcomes in our paper.

\section{References}

[1] A. Bartoszewicz, M. Bienias, S. Głąb, and T. Natkaniec, "Algebraic structures in the sets of surjective functions," Journal of Mathematical Analysis and Applications, vol. 441, no. 2, pp. 574585, 2016.

[2] R. M. Aron, L. B. Gonález, D. M. Pellegrino, and J. B. S. Sepúlveda, Lineability: The Search for Linearity in Mathematics, Monographs and Research Notes in Mathematics, CRC Press, Boca Raton, FL, USA, 2016.

[3] G. Peano, "Sur une courbe, qui remplit toute une aire plane," Mathematische Annalen, vol. 36, no. 1, pp. 157-160, 1890.

[4] N. G. Albuquerque, "Maximal lineability of the set of continuous surjections," Bulletin of the Belgian Mathematical Society Simon Stevin, vol. 21, no. 1, pp. 83-87, 2014.

[5] L. Bernal-Gonzállez, M. C. Calderón-Moreno, and J. A. PradoBassas, "The set of space-filling curves: topological and algebraic structure," Linear Algebra and its Applications, vol. 467, pp. 57-74, 2015.

[6] A. Bartoszewicz and S. Głąb, "Strong algebrability of sets of sequences and functions," Proceedings of the American Mathematical Society, vol. 141, no. 3, pp. 827-835, 2013.

[7] P. Billingsley, Probability and Measure, John Wiley \& Sons, New York, NY, USA, 1995.

[8] J. A. Conejero, M. Fenoy, M. Murillo-Arcila, and J. B. SeoaneSepúlveda, "Lineability within probability theory settings," Revista de la Real Academia de Ciencias Exactas, Físicas y Naturales. Serie A. Matematicas. RACSAM, vol. 111, no. 3, pp. 673-684, 2017.

[9] J. M. Stoyanov, Counterexamples in probability, Wiley Series in Probability and Statistics, John Wiley \& Sons, Ltd., Chichester, 1997.

[10] K. Ciesielski and M. Laczkovich, "Strong Fubini properties for measure and category," Fundamenta Mathematicae, vol. 178, no. 2, pp. 171-188, 2003.

[11] C. Freiling, "Axioms of symmetry: throwing darts at the real number line," The Journal of Symbolic Logic, vol. 51, no. 1, pp. 190-200, 1986.

[12] H. Friedman, "A consistent Fubini-Tonelli theorem for nonmeasurable functions," Illinois Journal of Mathematics, vol. 24, no. 3, pp. 390-395, 1980.

[13] W. Sierpiński, "Sur les rapports entre l'existence des intégrales $\int_{0}^{1} f(x, y) d x, \int_{0}^{1} f(x, y) d y$ et $\int_{0}^{1} d x \int_{0}^{1} f(x, y) d y$, , $P W N$, vol. 2, pp. 341-345, 1975.

[14] L. Bukovský, The structure of the real line, vol. 71, Mathematics Institute of the Polish Academy of Sciences, 2011. 


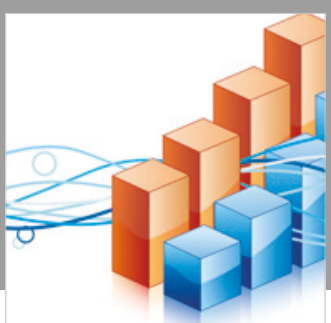

Advances in

Operations Research

\section{-n-m}
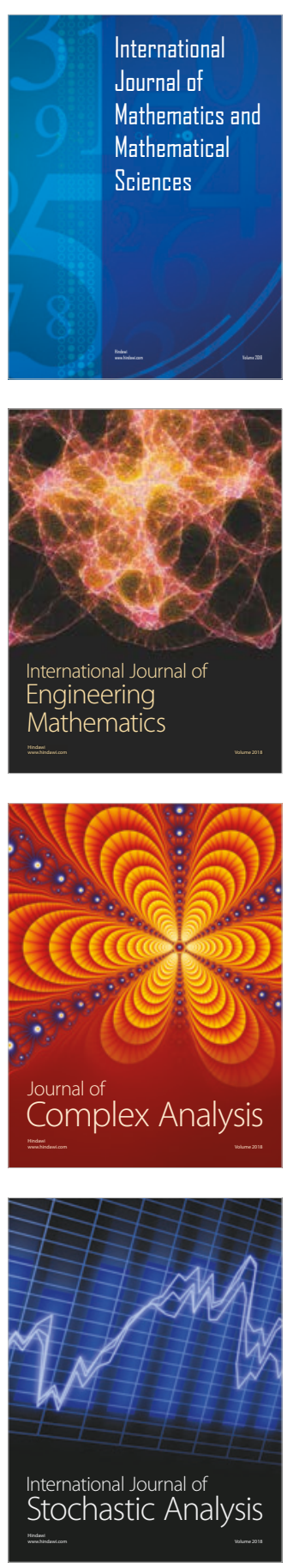
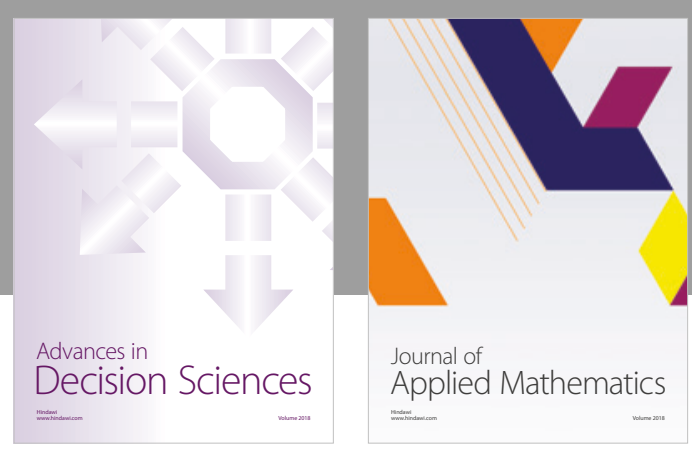

Journal of

Applied Mathematics
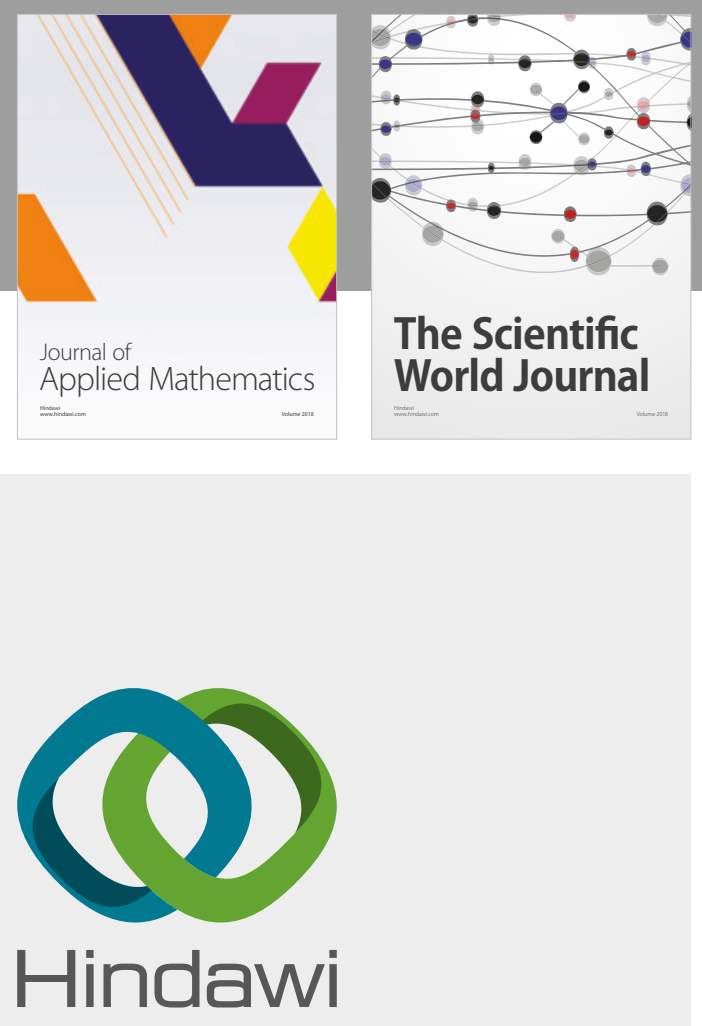

Submit your manuscripts at

www.hindawi.com

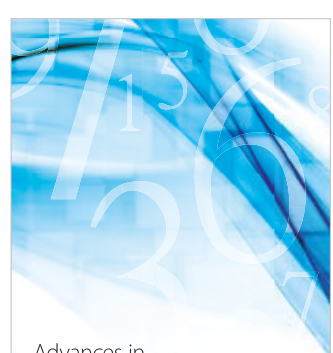

Advances in
Numerical Analysis
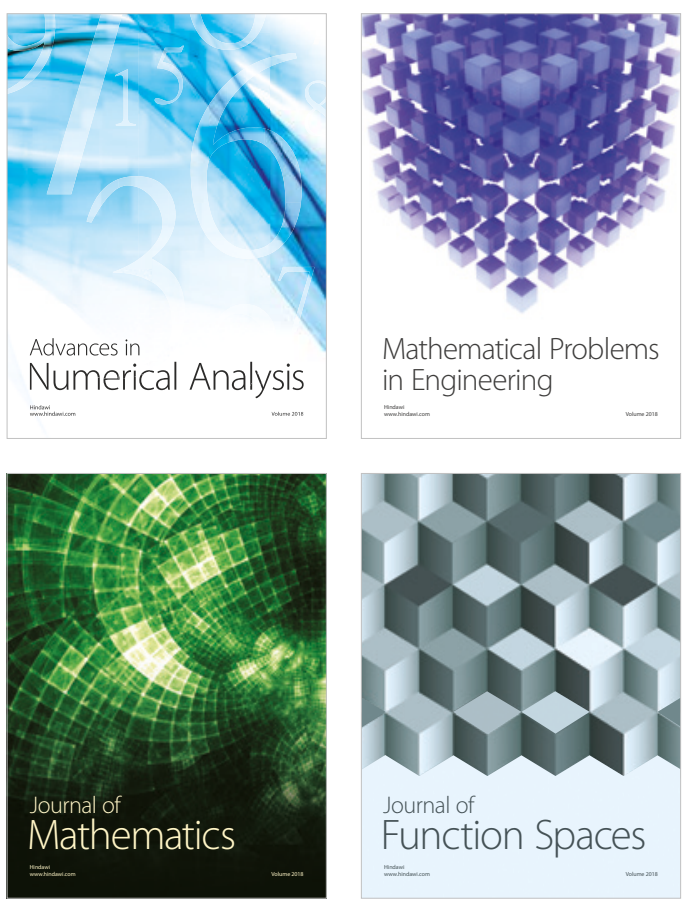

Mathematical Problems in Engineering

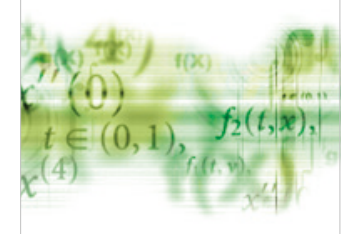

International Journal of

Differential Equations

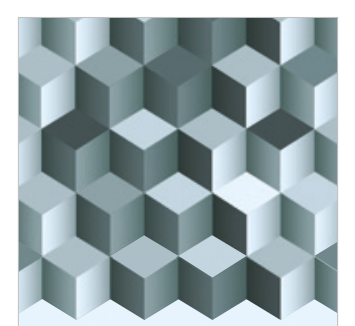

Journal of

Function Spaces

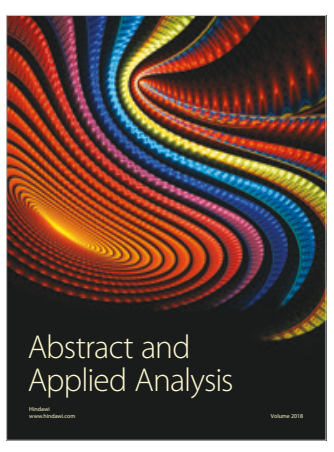

The Scientific

World Journal

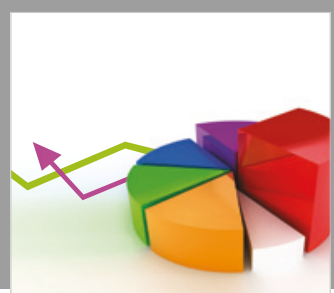

Journal of

Probability and Statistics
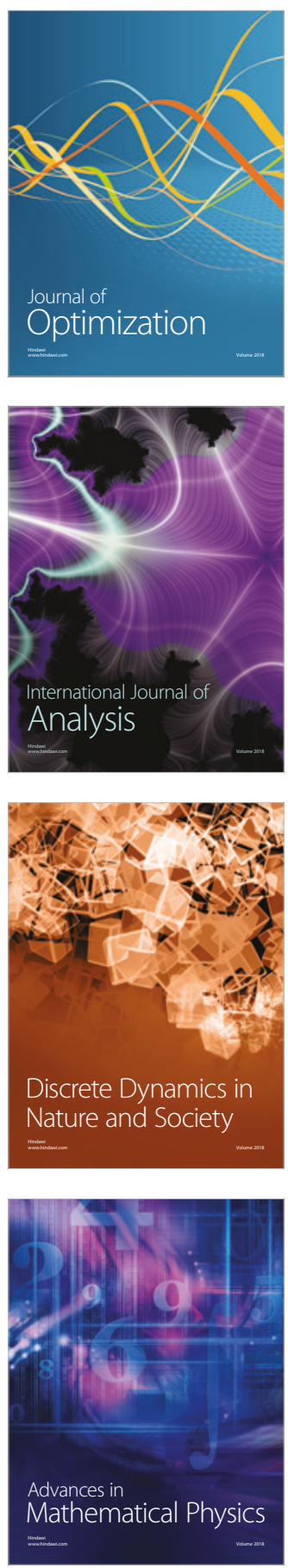